\title{
Largest and smallest minimal percolating sets in trees
}

\author{
Eric Riedl \\ Harvard University \\ Department of Mathematics \\ ebriedl@math.harvard.edu
}

Submitted: Sep 2, 2010; Accepted: Mar 21, 2012; Published: Mar 31, 2012

\begin{abstract}
Originally introduced by Chalupa, Leath and Reich for use in modeling disordered magnetic systems, $r$-bootstrap percolation is the following deterministic process on a graph. Given an initial infected set, vertices with at least $r$ infected neighbors are infected until no new vertices can be infected. A set percolates if it infects all the vertices of the graph, and a percolating set is minimal if no proper subset percolates. We consider minimal percolating sets in finite trees. We show that if $A$ is a minimal percolating set on a tree $T$ with $n$ vertices and $\ell$ vertices of degree less than $r$ (leaves in the case $r=2$ ), then $\frac{(r-1) n+1}{r} \leq|A| \leq \frac{r n+\ell}{r+1}$. Moreover, we show that the difference between the sizes of a largest and smallest minimal percolating sets is at most $\frac{(r-1)(n-1)}{r^{2}}$. Finally, we describe $O(n)$ algorithms for computing the largest (for $r=2$ ) and smallest (for $r \geq 2$ ) minimal percolating sets.
\end{abstract}

\section{Introduction}

In this paper we study the following deterministic process on a graph $G$, known as $r$ neighbor bootstrap percolation. Let $A_{0}=A$ be a subset of the vertices of $G$, and for each $t \geq 1$, let $A_{t}=A_{t-1} \cup B_{t}$, where $B_{t}$ is the set of vertices with at least $r$ neighbors in $A_{t-1}$. We write $\langle A\rangle=\cup_{t=0}^{\infty} A_{t}$, and say that $A$ percolates if $\langle A\rangle=V(G)$. We think of the process as modeling the spread of infection, with $A$ the set of initially infected vertices and $\langle A\rangle$ the set of eventually infected vertices. A set $A$ is a minimal percolating set if $A$ percolates but no proper subset of $A$ percolates. Note that such sets must exist, as $\langle G\rangle=G$. It turns out that minimal percolating sets are not necessarily all the same size. In this paper, we concern ourselves with the following questions: given a certain finite graph, what are the sizes of the largest and smallest minimal percolating sets? What bounds can we prove on the sizes of these sets? Can we provide examples of such sets? This paper considers these questions for trees.

Bootstrap percolation was introduced in 1979 by Chalupa, Leath, and Reich in [9] who were motivated by applications to disordered magnetic systems. It can be used to 
model many different real-world phenomena, including magnetic matericals, fluid flow in rocks, and computer storage systems. For more details on applications of bootstrap percolation, see the survey article by Adler and Lev [1]. Theoretical work on $r$-bootstrap percolation has focused mostly on variants of the following probabilistic question. Given a graph $G$, choose a randomly infected set of of vertices $A$ by letting each $v \in G$ be in $A$ independently with probability $p$. Then what is $p_{c}(G, r)=\inf \{p \mid \mathbb{P}(A$ percolates $) \geq 1 / 2\}$ ? Aizenman and Lebowitz and Cerf and Cirillo did foundational work for the problem on grids $[n]^{d}$ in $[2,7]$, and Cerf and Manzo [8] proved that

$$
p_{c}\left([n]^{d}, r\right)=\Theta\left(\frac{1}{\log ^{(r-1)} n}\right)^{d-r+1},
$$

where $\log ^{(r)}(x)$ is $\log (\log (\cdots \log (x)))$ ( $r$ times). Holroyd [10] found more precise estimates for $r=2, d=2$ and Balogh, Bollobás, Duminil-Copin and Morris [3,4] found precise asymptotics for all $r$ and $d$. Balogh, Bollobás and Morris also found asymptotic results for $d \gg \log (n)$, and $n$ and $d$ both increasing [5]. Balogh, Peres and Pete found $p_{c}$ for infinite trees in [6].

Write $m(G, r)$ for the size of a smallest minimal percolating set, and $E(G, r)$ for the size of a largest minimal percolating set under $r$-bootstrap percolation. In this paper we bound $m$ and $E$. As seen in many cases (such as [11]), information about the size and structure of minimal percolating sets can often be both useful in answering the original probabilistic percolation questions and interesting in terms of gaining insight into the percolation process. Minimal percolating sets are crucial to understanding the structure of percolating sets, as any percolating set necessarily contains a minimal percolating set. Bounding the maximum and minimum sizes of minimal percolating sets is a basic first step in investigating minimal percolating sets. Moreover, studying $E$ and $m$ gives a sense of how badly the greedy algorithm could fail in minimizing the number of sites required for percolation.

Less work has been done on the extremal problem than on the probabilistic problem. It is a folk-lore fact that for $r=2$, the smallest minimal percolating set in the $n \times n$ grid has size $n$. Pete [12] computes $m\left([n]^{d}, r\right)$ up to a constant factor for every fixed $d$ and $r$, and proves more precise estimates in special cases. Morris [11], answers a question of Bollobás and finds asymptotic bounds for the size of a largest minimal percolating set in $[n]^{2}$, showing that it lies between $\frac{4 n^{2}}{33}$ and $\frac{n^{2}}{6}$. We investigate $E$ and $m$ for finite trees. Since trees are a diverse family of graphs, it is difficult to find a simple formula for $E$ and $m$. Indeed, there is no known simple numerical characterization of trees which can be used to determine the sizes of their minimal percolating sets. Candidates such as number of vertices or degree sequence simply do not carry enough information. For example, the graphs in Figure 1 have the same number of vertices and the same degree sequence but different smallest minimal percolating set sizes.

Our main results are bounds on the sizes of minimal percolating sets and $O(n)$ algorithms for computing the sizes of minimal percolating sets. More specifically, in Section 2 we use an edge-counting technique to obtain three bounds on sizes of minimal percolating sets, which when combined give the following. 

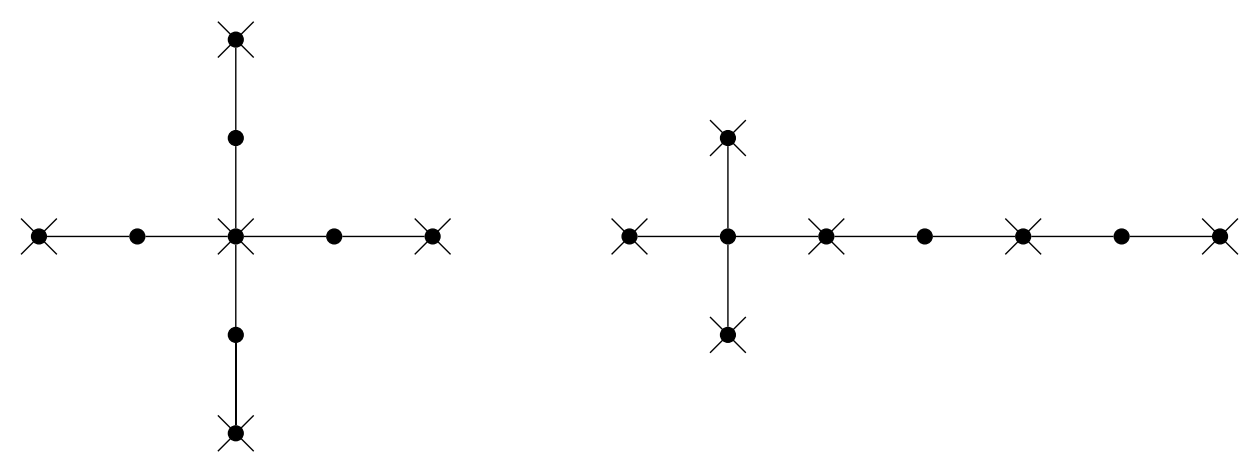

Figure 1: Smallest minimal percolating sets in two trees with the same numbers of vertices and degree sequences but different sizes of the smallest minimal percolating set.

Main Theorem. Let $T$ be a tree with $n$ vertices and $\ell$ leaves. Then

$$
\frac{(r-1) n+1}{r} \leq m(T, r) \leq E(T, r) \leq \frac{r n+\ell}{r+1}
$$

and

$$
E(T, r)-m(T, r) \leq \frac{(r-1)(n-1)}{r^{2}}
$$

We exhibit infinite families for which all of the bounds are sharp except for the bound on $E(T, r)-m(T, r)$ with $r=2$; in this case we exhibit an infinite family of graphs for which $E(T, r)-m(T, r)$ is within 1 of the bound. We prove our main theorem in three parts. Proposition 3 is the lower bound on $m(T, r)$, Theorem 4 is the upper bound on $E(T, r)$ and Theorem 5 is the bound on $E(T, r)-m(T, r)$. In Section 3 we describe $O(n)$ algorithms for computing $m(T, r)$ for all $r \geq 2$ and $E(T, 2)$.

\section{Bounds}

In this section we give some bounds on the maximum and minimum size of a minimal percolating set, as well as the difference between them. Throughout we let $|T|=n$ unless otherwise stated. We give three main bounds. First, we show $m(T, r) \geq \frac{(r-1) n+1}{r}$. Note that this is sharp for a complete $r$-ary tree. Second, if $\ell$ is the number of vertices of degree less than $r$, we show $E(T, r) \leq \frac{r n+\ell}{r+1}$. Finally, we show $E(T, r)-m(T, r) \leq \frac{(r-1)(n-1)}{r^{2}}$.

In order to prove our results, we need to define the notion of a wasted edge. Let $G$ be a graph and fix a percolating set $A$ on $G$. Intuitively, some of the edges of $G$ are "used," and are necessary for $A$ to percolate, whereas others are "wasted," because we can remove them and the graph will still percolate.

We now make this intuitive notion precise. We give each edge of our graph one of two designations: wasted or used, and we give each used edge a direction. Start with a set $A$ of initially infected vertices. Each time a new vertex $v$ is infected in the percolation process, choose $r$ of the edges connecting $v$ to infected vertices and call them used, directing 


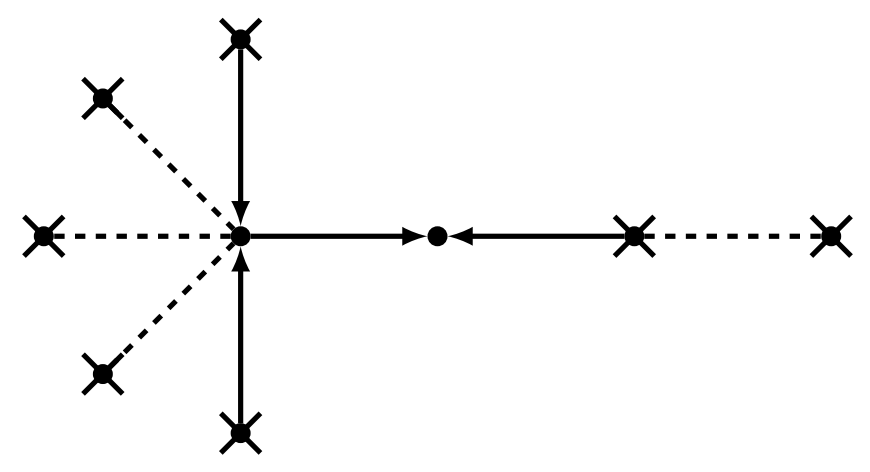

Figure 2: A choice of wasted edges in a broom.

them toward $v$. Continue this process until all of the vertices are infected. Call all of the undirected edges wasted. Note that it is impossible to give an edge more than one direction, as directions are only given to edges incident to uninfected vertices, and once an edge is given a direction, both of the vertices it is incident to are infected. If $G$ is a graph and $A$ a percolating set, then given a choice of wasted edges, $A$ will percolate in $G$ minus the wasted edges. See Figure 2 for an example of a choice of wasted edges in a "broom." Let $w$ be the number of wasted edges. Now, in most cases there is freedom involved in choosing wasted and used edges, and it is not immediately clear that $w$ is well-defined. The next proposition shows that it is.

Proposition 1. Let $G$ be a graph with $e(G)$ edges and let $A$ be a percolating set in $G$. Then $w$, the number of wasted edges, is well defined for any choice of used edges, and

$$
w=e(G)-r(|G|-|A|) .
$$

Proof. Each vertex $v$ of $G \backslash A$ is infected by exactly $r$ used edges directed toward $v$. Since each edge is given only one direction, we know that there are exactly $r(|G|-|A|)$ used edges. Hence, there are $e(G)-r(|G|-|A|)$ wasted edges.

Corollary 2. If $T$ is a tree with $|T|=n$, and $w$ is the number of edges wasted for a percolating set $A$, then

$$
w=-(r-1) n-1+r|A| .
$$

As an immediate application of the notion of wasted edges, we deduce a lower bound on $m(T, r)$ for trees.

Proposition 3. Let $T$ be a tree, $|T|=n>2$. Then

$$
m(T, r) \geq \frac{(r-1) n+1}{r}
$$

with equality if and only if there exists a minimal percolating set with no wasted edges. 
Note that this bound is sharp. For example, if $r=2$, paths with an odd number of vertices and complete binary trees both have $m(T)=\frac{n+1}{2}$. For $r>2$, complete $(r-1)$-ary and $r$-ary trees have $m(T)=\frac{(r-1) n+1}{r}$. Indeed, for complete $r$-ary trees, $m(T)=E(T)$, so this also gives a sharp lower bound for $E(T, r)$.

Proof. The number of wasted edges is clearly nonnegative:

$$
w=-(r-1) n-1+r|A| \geq 0 .
$$

Thus,

$$
r|A| \geq(r-1) n+1
$$

which gives

$$
|A| \geq \frac{(r-1) n+1}{r} .
$$

Note that while we are only concerned with trees in this paper, the idea of the above proof of Proposition 3 works for any graph $G$, giving $m(G, r) \geq|G|-\frac{e(G)}{r}$.

We now turn to upper bounds on the size of minimal percolating sets. For $r>2$, paths are an example of trees for which $E(T, r)=m(T, r)=|T|$. For $r=2$, we have trivially that $E(T, 2) \leq|T|-1$ for $|T|>2$, as any tree of size at least 3 must have a vertex of degree at least 2, so it is impossible for $V(G)$ to be a minimal percolating set. However, for stars we have $E(T, 2)=m(T, 2)=|T|-1$. Hence, the sharp upper bounds which hold for all trees are not particularly interesting. Note, however, that these examples all have lots of vertices of degree less than $r$, which forced the sizes of minimal percolating sets to be large. If we keep track of how many such vertices we have, we can get a more interesting bound.

Theorem 4. Let $G$ be a graph with $\ell$ vertices of degree less than $r,|G|=n$. Then

$$
E(G, r) \leq \frac{r n+\ell}{r+1}
$$

Proof. The key observation is that if $A$ is a minimal percolating set, and $v \in A$ of degree at least $r$, then $v$ must be incident with at least one used edge. Since a used edge is incident with at most one vertex in $A$, this means that the number of used edges is at least the number of vertices in $A$ of degree greater than or equal to $r$. Thus,

$$
r(n-|A|) \geq|A|-\ell
$$

or

$$
|A| \leq \frac{r n+\ell}{r+1}
$$




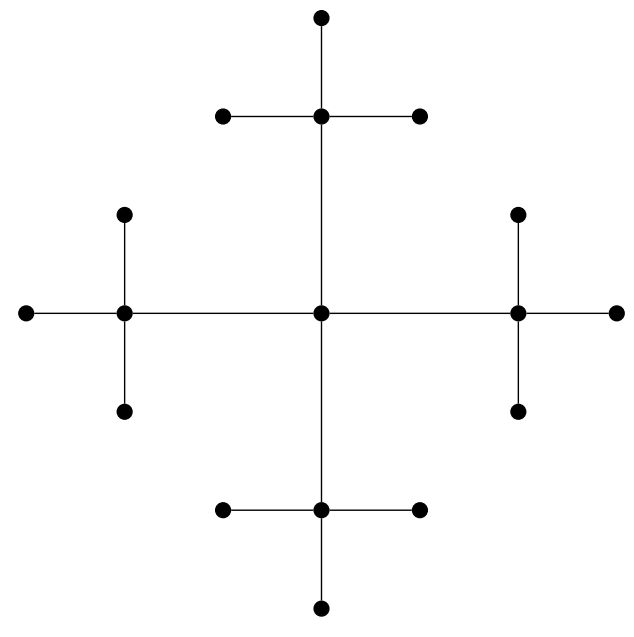

Figure 3: Example of graph with maximum $\frac{E(T, 4)-m(T, 4)}{n-1}$.

The bound in Theorem 4 is sharp (up to integer parts) for paths, stars, and brooms (paths with some number of pendant edges attached to one of the leaves) for $r=2$. For $r \geq 3$, the result is sharp for paths, stars (up to integer parts) and for graphs obtained by attaching $r-1$ pendant edges to each leaf in a star with $r$ leaves.

We have just given bounds on both $m(T, r)$ and $E(T, r)$ separately. We now bound their difference. Since the possible difference in sizes will obviously grow with $|T|$, we consider the largest possible difference as a function of $n=|T|$.

Theorem 5. For any tree $T$ with $|T|=n>1$ we have

$$
E(T, r)-m(T, r) \leq \frac{(r-1)(n-1)}{r^{2}}
$$

Before embarking on the proof of Theorem 5, we give some examples to show that the above bound is sharp. Construct the following tree $T$. Start with a vertex $c$. Then attach $r$ vertices to $c$. Finally, attach $r-1$ vertices to each new vertex. For $r=2$ this is simply $P_{5}$. Now, for these trees, we have $E(T, r)=r^{2}$ (given by the set $T \backslash c$ ) and $m(T, r)=r(r-1)+1$ (given by the set consisting of $c$ and the leaves of $T$ ). Thus, $\frac{E(T, r)-m(T, r)}{|T|-1}=\frac{r-1}{r^{2}}$. See Figure 3 .

The next natural question to ask is whether there is an infinite family of graphs that have $\frac{E(T, r)-m(T, r)}{|T|-1}=\frac{r-1}{r^{2}}$. For $r>2$, the answer to this question is yes. We construct our family inductively. Suppose $T_{1}$ and $T_{2}$ are two trees with maximal $E\left(T_{i}, r\right)-m\left(T_{i}, r\right)$. Let $w_{1}$ be a leaf of $T_{1}$ and let $w_{2}$ be a leaf of $T_{2}$. Then the tree obtained by identifying $w_{1}$ and $w_{2}$ into one vertex (of degree 2 ) will be another tree which satisfies the equality.

For $r=2$, the above construction will not work. For $r>2$, vertices of degree 2 "behave like leaves" in the sense that they must be infected in any minimal percolating set, but for $r=2$, this is certainly not the case. We do not know of an infinite family of graphs which satisfy the equality for $r=2$. However, we can display an infinite family of 


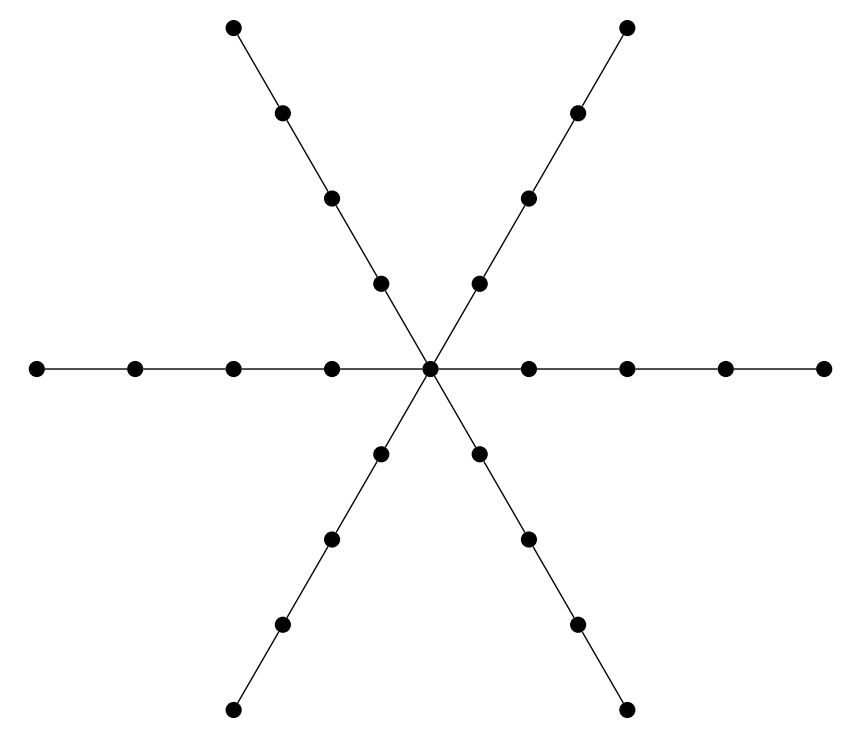

Figure 4: Example of $6 P_{5}$ 's joined together at their leaves.

graphs which get arbitrarily close to the (perhaps more natural) bound $\frac{E(T, 2)-m(T, 2)}{n}<\frac{1}{4}$. Take $k P_{5}$ 's and choose a leaf of each $P_{5}$. Glue all of the $P_{5}$ 's togehter by identifying these leaves into a single vertex $v$. Then the resulting graph will have $E(T, 2)=3 k$ (the minimal percolating set consists of all leaves, neighbors of leaves and neighbors of $v$ ) and $m(T, 2)=2 k+1$ (the minimal percolating set consists of $v$, all leaves, and all vertices of distance 2 from $v$ ). Thus, for this tree $\frac{E(T, 2)-m(T, 2)}{n}=\frac{k-1}{4 k+1}$, which gets arbitrarily close to $\frac{1}{4}$ as $k$ gets large.

In proving Theorem 5, we first show the result for a large class of trees, then extend the result to all trees. Before proceeding, we introduce some terminology. Recall that $\langle A\rangle$ is the set of vertices eventually infected by an initial set $A$. In case the ambient graph is not obvious, we write $\langle A\rangle_{G}$ for the set of vertices in $G$ eventually infected by $A$ under the percolation process. A vertex $v$ is said to be traceable back to leaves if $v \in\langle L\rangle$, where $L$ is the set of leaves. Note that leaves are trivially traceable back to leaves. We now prove a crucial lemma.

Lemma 6. Let $T$ be a tree with $|T|>2$ such that no nonleaf is traceable back to leaves, and every nonleaf has degree at least $r$. Let $A$ be a minimal percolating set. Then a fraction of at most $\frac{r-1}{r}$ of the edges of $T$ are wasted. That is, $w \leq \frac{(n-1)(r-1)}{r}$.

Proof. First, we show that we can choose a set of wasted edges such that every wasted edge is incident with a nonleaf in $A$. Suppose we are given a choice of wasted edges such that $k$ wasted edges that are incident with a nonleaf in $A$, with $k$ strictly less than the total number of wasted edges. We construct a choice of wasted edges such that $k+1$ wasted edges are incident with a nonleaf in $A$.

Let $e$ be a wasted edge that is not incident to a nonleaf in $A$. Since $n>2$, e will be incident with at least one nonleaf, so let $v$ be a nonleaf incident with $e$. Let $\Omega$ be the set 
of directed paths of used edges from nonleaf vertices in $A$ to $v$. Since $v$ is not traceable back to leaves, $\Omega$ is non-empty, so choose a path $\left(v_{0}, \cdots, v_{d}\right) \in \Omega$, with $v_{0}$ a nonleaf in $A, v_{d}=v$. We now exhibit a choice of wasted edges with $k+1$ wasted edges incident with a nonleaf in $A$ : take the original choice of wasted edges, except designate $e$ as used and direct it toward $v$, reverse the direction of every edge $\overline{v_{i} v_{i+1}}$ for $1 \leq i \leq d-1$ and designate $\overline{v_{0} v_{1}}$ as wasted. This will be a legal choice of wasted and used edges, and since $v_{0}$ is a nonleaf in $A$, we will have exactly one more wasted edge which is incident with a nonleaf in $A$.

Now, any vertex $v \in A$ is adjacent to at most $r-1$ wasted edges, since if $v$ were adjacent to $r$ or more, $A \backslash v$ would percolate, which contradicts the minimality of $A$. Thus, if $B \subset A$ is the set of nonleaves, then

$$
w \leq(r-1)|B|
$$

Let $u$ be the number of used edges. Then every vertex of $B$ is incident with at least one used edge, and since edges between two elements of $A$ cannot be used, we have

$$
u \geq|B|
$$

Thus $w \leq(r-1) u$. Now, since $\frac{u+w}{n-1}=1$, we have $w \leq \frac{(n-1)(r-1)}{r}$.

Now we use the above result to prove an upper bound for $E(T, r)$ for the specific class of trees to which we have restricted ourselves.

Proposition 7. Let $T$ be a tree with no vertices traceable back to leaves and with the property that every nonleaf has degree at least $r$. Then

$$
E(T, r) \leq \frac{\left(r^{2}-1\right) n+1}{r^{2}}
$$

Proof. Let $A$ be a minimal percolating set with $|A|=E(T, r)$. From Lemma 6 , we have

$$
r w \leq(n-1)(r-1) .
$$

Using Proposition 1 we have

$$
\begin{gathered}
-r(r-1) n-r+r^{2}|A| \leq n r-n-r+1 \\
|A| \leq \frac{\left(r^{2}-1\right) n+1}{r^{2}} .
\end{gathered}
$$

Corollary 8. For trees $T$ with every nonleaf having degree at least $r$ and no nonleaf vertices traceable back to leaves,

$$
E(T, r)-m(T, r) \leq \frac{(r-1)(n-1)}{r^{2}}
$$


Proof. Simply subtract the inequalities from Proposition 3 and Proposition 7.

Proof of Theorem 5. Now, we finally extend our result to general trees $T$ using Corollary 8. First, we dispose of the condition that no nonleaves are traceable back to leaves. Suppose, to get a contradiction, that $T$ is a smallest tree with $E(T, r)-m(T, r)>$ $\frac{(r-1)(|T|-1)}{r^{2}}$ such that every nonleaf of $T$ has degree at least $r$. Then (since by Corollary 8 any such tree $T$ must have a vertex traceable back to leaves) there is a vertex $v \in T$ with at least $r$ pendant edges. Let $L$ be the set of leaves attached to $v$. Let $T_{1}^{\prime}, \ldots, T_{k}^{\prime}$ be the connected components of $T \backslash(v \cup L)$. Let $T_{i}$ be $T_{i}^{\prime}$ with one leaf $w_{i}$ adjoined to the unique neighbor of $v$ in $T_{i}$. Note that all nonleaves in $T_{i}$ have degree at least $r$.

Now, there is a canonical bijection between minimal percolating sets on the disjoint union of the $T_{i}$ 's and minimal percolating sets of $T$. Take a minimal percolating set $A$ of $T$. Note that $v \notin A$. For each $i$, set $A_{i}=\left(A \cap T_{i}\right) \cup w_{i}$. Then each $A_{i}$ will be a minimal percolating set of $T_{i}$, and similarly, given $A_{i}$ which are minimal percolating sets in the $T_{i}, L \cup \cup_{i}\left(A_{i} \backslash w_{i}\right)$ will be a minimal percolating set in $T$. Thus, we have $|T|=\sum_{i}\left(\left|T_{i}\right|-1\right)+1+|L|$, while $E(T, r)-m(T, r)=\sum_{i}\left(E\left(T_{i}, r\right)-m\left(T_{i}, r\right)\right)$. Hence,

$$
\begin{aligned}
\frac{E(T, r)-m(T, r)}{|T|-1}= & \frac{\sum_{i}\left(E\left(T_{i}, r\right)-m\left(T_{i}, r\right)\right)}{\sum_{i}\left(\left|T_{i}\right|-1\right)+|L|}<\frac{\sum_{i}\left(E\left(T_{i}, r\right)-m\left(T_{i}, r\right)\right)}{\sum_{i}\left(\left|T_{i}\right|-1\right)} \\
& \leq \max _{i}\left\{\frac{E\left(T_{i}, r\right)-m\left(T_{i}, r\right)}{\left|T_{i}\right|-1}\right\}
\end{aligned}
$$

Thus, one of the $T_{i}$ also satisfies $E\left(T_{i}, r\right)-m\left(T_{i}, r\right)>\frac{(r-1)\left(\left|T_{i}\right|-1\right)}{r^{2}}$. However, this contradicts minimality of $T$, as $\left|T_{i}\right|<|T|$. Thus, our result holds for all trees in which every nonleaf has degree at least $r$.

Now we dispose of the condition that every nonleaf of $T$ has degree at least $r$, so this paragraph applies only for $r>2$. To get a contradiction, find a smallest tree $T$ such that $E(T, r)-m(T, r)>\frac{(r-1)(|T|-1)}{r^{2}}$. By the result of the previous paragraph, we can find a vertex $v$ of $T$ of degree $1<d<r$. Thus, $v$ will have to be infected in any minimal percolating set. Let $T_{1}, \ldots, T_{n}$ be the connected components of $T \backslash v$ with an extra leaf $w_{i}$ added to the vertex connected to $v$.

As above, there is a canonical bijection between minimal percolating sets in the disjoint union of the $T_{i}$ 's and minimal percolating sets of $T$. Namely, given a minimal percolating set $A$ of $T,\left(A \cap T_{i}\right) \cup w_{i}$ will be a minimal percolating set for each of the $T_{i}$, and if $A_{i}$ are minimal percolating sets for each of the $T_{i}, v \cup \cup_{i}\left(A_{i} \backslash w_{i}\right)$ will be a minimal percolating set of $T$. Now, consider the quantity $\frac{E(T, r)-m(T, r)}{|T|-1}$. We have $E(T, r)-m(T, r)=$ $\sum_{i}\left(E\left(T_{i}, r\right)-m\left(T_{i}, r\right)\right)$. Moreover, $|T|=\sum_{i}\left(\left|T_{i}\right|-1\right)+1$. Thus, we have

$$
\frac{E(T, r)-m(T, r)}{|T|-1}=\frac{\sum_{i}\left(E\left(T_{i}, r\right)-m\left(T_{i}, r\right)\right)}{\sum_{i}\left(\left|T_{i}\right|-1\right)} \leq \max _{i}\left\{\frac{E\left(T_{i}, r\right)-m\left(T_{i}, r\right)}{\left|T_{i}\right|-1}\right\} .
$$

Thus, one of the $T_{i}$ also satisfies $E\left(T_{i}, r\right)-m\left(T_{i}, r\right)>\frac{(r-1)\left(\left|T_{i}\right|-1\right)}{r^{2}}$. This contradicts the minimality of $T$. Hence, our result holds for all trees. 


\section{Algorithms}

In this section, we describe algorithms to compute $m(T, r)$ and $E(T, 2)$. The basic idea of the algorithms is to recursively solve the problem by finding and modifying certain specific subtrees. Note that in general, extremal (that is, largest and smallest) minimal percolating sets are not unique, and if we run our algorithm in a slightly different order we could end up with a different extremal minimal percolating set, although the size will of course be the same.

Before presenting the algorithms, we need to define a few terms. We say that $H$ is a trailing path (or a trailing $P_{k}$ ) if $H$ is a path of length $k-1$ and is connected to $G \backslash H$ by a single edge going from one of the ends of the path to the rest of the graph. We say that $H$ is a trailing star if $H$ is a $K_{1, \ell}$ with $H$ connected to $G \backslash H$ by a single edge from the center vertex of the star $H$.

We will also need the notion of a pseudo-star. We say that a tree $H$ with center vertex $v$ is a pseudo-star with center $v$ if every vertex of $H$ has distance at most 2 from $v$. Define a trailing pseudo-star to be a subtree that is a pseudo-star connected to the rest of $G$ by a single edge from the center $v$ of the pseudo-star. Note that stars are pseudo-stars, and trailing stars are trailing pseudo-stars. A straight pseudo-star is a pseudo-star for which every vertex except the center has degree at most 2, while a branched pseudo-star is any pseudo-star which is not straight.

We describe two different algorithms, mSet and ESet, for computing $m(T, r)$ and $E(T, r)$ respectively. Both of the algorithms presented consist of the same basic steps. Note Step 1 is unnecessary in the case where $r=2$, and hence is unnecessary for our algorithm ESet.

Step 1 Repeatedly perform the reduction procedure (to be described) until every vertex has degree 1 or at least $r$.

Step 2 Identify a trailing star or pseudo-star that can be reduced (we will describe below which trailing subgraphs can be reduced in which instances).

Step 3 Form the graph $T^{\prime}$ by modifying the trailing star, pseudo-star or path (in a manner that will be described below).

Step 4 Set $A^{\prime}=\operatorname{mSet}\left(T^{\prime}, r\right)$ or $A^{\prime}=\operatorname{ESet}\left(T^{\prime}, r\right)$.

Step 5 Modify $A^{\prime}$ (in a manner that will be described below) to obtain $A$, a smallest or largest minimal percolating set of $T$.

Step 6 Output $A$.

Steps 4 and 6 are self-explanatory. Steps 2, 3 and 5 depend on the specifics of $r$ and whether or not we are computing $m(T, r)$ or $E(T, r)$, and we elaborate on them in detail below. The purpose of Step 1 is to ensure that every non-leaf has degree at least $r$, and it is simple enough that we describe it now. 
Step 1 is performed as follows. Iterate through each vertex $v$ of degree less than $r$. Let $T_{i}^{\prime}$ be the connected components of $T \backslash v$. Let $T_{i}$ be $T_{i}^{\prime} \cup w_{i}$, where $w_{i}$ is a single leaf attached to the unique neighbor of $v$ in $T_{i}^{\prime}$. Perform the algorithm on all of the $T_{i}$, obtaining minimal percolating sets $A_{i} \cup w_{i}$. Let $A=v \cup \cup A_{i}$ be the output of the algorithm.

\section{$3.1 m(T, r)$}

Because the proofs of the algorithms are often long and a bit tedious, we sketch many of them. We start by elaborating on step 2. When computing $m(T, r)$, we reduce by identifying trailing stars, so in order to implement step 2, we need to know that we can always identify trailing stars, and prove that we can do so efficiently.

Lemma 9. Every tree has a trailing star.

Proof. Find a longest path in $T$, with $v$ the second-to-last vertex of the path. Then $v$ will be the center of a trailing star.

The above proof suggests an algorithm for identifying trailing stars, and this is essentially the method we adopt. However, because the proof of $O(n)$ runtime is somewhat intricate, we defer discussion of this until after describing Steps 3 and 5 .

Steps 3 and 5 divide into the following two cases, depending on the number of leaves of the trailing star. Let $v$ be the center vertex of the trailing star identified in Step 2, and $L$ the set of leaves of the trailing star.

Case A: $|L|<r$. Note that because of Step 1, this implies $|L|=r-1$. In the case of $r=2$, this is simply a trailing $P_{2}$. When performing Step 3 in this case, we set $T^{\prime}=T \backslash(v \cup L)$. When performing Step 5 in this case, we set $A=A^{\prime} \cup L$.

Case B: $|L| \geq r$. When performing Step 3 in this case, we set $T^{\prime}=T \backslash L$. When performing Step 5 in this case, we set $A=A^{\prime} \cup L \backslash v$. Note that $v$ is a leaf in $T^{\prime}$, so $v$ will always be in $A^{\prime}$.

This gives a complete description of how to perform our algorithm for $m(T, r)$. Because the proof that our algorithm works is tedious and relatively intuitive, we merely sketch the proofs. We start with Case B.

Lemma 10. Given a graph $T$ with a trailing star $v \cup L$ ( $v$ the center, $L$ the leaves) with $|L| \geq r$, and given a smallest minimal percolating set $A^{\prime}$ for $T^{\prime}=T \backslash L$, we then have $A^{\prime} \cup L \backslash v$ is a smallest minimal percolating set for $T$.

Proof. In any minimal percolating set of $T$, every vertex of $L$ must be infected. The vertices of $L$ alone are sufficient to infect $v$. Thus, when constructing a minimal percolating set of $T$, we can "imagine" that $v$ is a leaf and that the vertices of $L$ do not exist.

The proof for Case A requires one auxiliary lemma. 
Lemma 11. If $T$ is a tree and $w$ is a leaf, then $m(T \backslash w, r) \leq m(T, r) \leq m(T \backslash w, r)+1$.

Proof. The first inequality holds because given a minimal percolating set $A$ of $T$, we can construct a percolating set $A^{\prime}$ of $T \backslash w$ of size $|A|$ by taking $A^{\prime}=A \cup v \backslash w$, where $v$ is the unique neighbor of $w$. The second inequality holds because given a minimal percolating set $A^{\prime}$ of $T \backslash v, A^{\prime} \cup v$ will be a percolating set of $T$.

Now we prove our algorithm works for Case A.

Lemma 12. If $T$ is a tree with trailing star $v \cup L$ ( $v$ the center, $L$ the set of leaves) and $|L|=r-1$, and if $A^{\prime}$ is a smallest minimal percolating set for $T^{\prime}=T \backslash(v \cup L)$, then $A^{\prime} \cup L$ is a smallest minimal percolating set for $T$.

Proof. We see immediately tha $A^{\prime} \cup L$ must percolate and that $L$ must be in any minimal percolating set, so our only concern is that there might be some minimal percolating set $A$ of $T$ which contains $v$ and has size strictly smaller than $\left|A^{\prime}\right|+|L|$. By Lemma 11 this is impossible, since $A \backslash L$ will be a minimal percolating set of $T \backslash L$.

Thus, we have shown that our algorithm does indeed produce a smallest minimal percolating set. Moreover, it is clear that each step reduces the number of edges by at least one, and the time taken at each step is linear in the number of edges removed, so the time spent on each step is linear in the number of edges. It remains to sketch how to quickly identify trailing stars.

Proposition 13. It is possible to efficiently identify trailing stars so that the algorithm mSet runs in $O(n)$ time.

Proof. Note that the total time taken to perform Step 1 is linear in the number of edges, so the total time spent on Step 1 is $O(n)$. Note also that Step 1 preserves the number of edges, so repeatedly applying Step 1 will not cause the problem to grow too much.

At the very beginning of the algorithm (before doing any recursion), make a list VertList of all of the non-leaves, and for each vertex in VertList, make a list of its non-leaf neighbors. This entire process grows linearly with the number of edges of the tree, and so will take $O(n)$ time. Note that a trailing star can be characterized as a non-leaf with exactly one non-leaf neighbor. Let CurrentVert be the first element of VertList.

Check if CurrentVert has only one non-leaf neighbor. If not, continue and set CurrentVert to be the next element in VertList. If so, we have identified a trailing star. Set CurrentVert to the next element of VertList, perform Steps 3-5 and update VertList and the lists of non-leaf neighbors. Now, in forming $T^{\prime}$, we remove precisely one non-leaf neigbor from a vertex CheckVert. Check to see if CheckVert is the center of a trailing star. If so, reduce, update VertList and the lists of non-leaf neighbors, and find the new CheckVert. If not, continue with VertList, checking to see if CurrentVert is a trailing star. After we have gone completely through VertList, we will be done. 


\section{$3.2 E(T, r)$}

In this section, we only consider the case $r=2$. As in our algorithm for $m(T, r)$, we begin by proving that we may always apply Step 2. However, in this case the three types of trailing subgraphs are trailing stars, trailing $P_{3}$ 's and trailing straight pseudo-stars.

Lemma 14. Every tree contains a trailing star, a trailing $P_{3}$ or a trailing straight pseudostar.

Proof. Let $v$ be the third-to-last vertex in a longest path in $T$. If the degree of $v$ is 2 , then either $v$ is part of a trailing $P_{3}$ or one of the neighbors of $v$ is the center of a trailing star. If the degree of $v$ is greater than 2, then $v$ is the center of a trailing pseudo-star. If the trailing pseudo-star is straight, then we are done. If it is branched, then one of the neighbors of $v$ will be a trailing star.

The proof that we can always efficiently identify trailing stars and psuedo-stars is similar enough to Proposition 13 that we omit it, although there are a few extra complications (we need to look for vertices that have only one non-leaf neighbor and that are not part of a trailing $P_{2}$ ).

Now we elaborate on how to perform Steps 3 and 5. There are four different cases.

Case A: Trailing star $v \cup L$, with $v$ the center vertex and $L$ the set of leaves. When performing Step 3 in this case, let $T^{\prime}=T \backslash L$. When performing Step 5 in this case, let $A=A^{\prime} \cup L \backslash v$.

Case B: Trailing straight pseudo-star with at least 2 leaves attached directly to the center vertex, with $v$ the center vertex of the pseudo-star, $S$ the rest of the vertices of the pseudo-star, and $L \subset S$ the set of leaves of the pseudo-star. When performing Step 3 in this case, let $T^{\prime}=T \backslash S$. When performing Step 5 in this case, let $A=A^{\prime} \cup L \backslash v$.

Case C: Trailing $P_{3}$, with $u$ the leaf, $v$ the unique neighbor of $u$ and $w$ the third vertex of the pseudo-star. When performing Step 3, let $T^{\prime}=T \backslash\{u, v, w\}$. When performing Step 5, let $A=A^{\prime} \cup\{u, v\}$.

Case D: Trailing straight pseudo-star with at most one leaf attached directly to the center vertex, with $v$ the center vertex, $S$ the rest of the pseudo-star, $L \subset S$ the set of leaves of the pseudo-star. When performing Step 3 in this case, let $T^{\prime}=T \cup u \backslash S$, where $u$ is a leaf attached directly to $v$. When performing step 5 , there are two cases. If $v \in A^{\prime}$, then let $A=A^{\prime} \cup X \backslash\{u, v\}$, where $X \subset S$ is a set containing $L$ which contains precisely two neighbors of $v$. (Thus, $X$ will be either $L$ union one non-leaf or it will be $L$ union two non-leaves.) If $v \notin A^{\prime}$, then let $A=A^{\prime} \cup Y \backslash u$, where $Y \subset S$ is a set containing $L$ which contains precisely one neighbor of $v$. (Thus, $Y$ will be either $L$, or it will be $L$ union one non-leaf.) Note that in general there will be many possible choices for $X$ and $Y$, and different choices will lead to different largest minimal percolating sets (although all such sets will of course have the same size). 
The proofs of Cases A and B are essentially the same as the proof of Case A in the mSet lemma, and so we omit them. Thus, it remains to prove the correctness of Cases $\mathrm{C}$ and D. We need the analogue of Lemma 11 (we only require the second inequality, but we prove both for completeness, and because the proof of the first is easier). We need some terminology for this Lemma. We say that a subtree $T^{\prime} \subset T$ is internally spanned by a set $A \subset T$ if $\left\langle A \cap T^{\prime}\right\rangle_{T^{\prime}}=T^{\prime}$.

Lemma 15. Let $T$ be a tree and $v$ a leaf. Then $E(T \backslash v, 2) \leq E(T, 2) \leq E(T \backslash v, 2)+1$.

Proof. The proof of both inequalities requires a careful analysis of what $U=T \backslash\langle A \backslash w\rangle$ looks like, where $A$ is a minimal percolating set and $w \in A$ is any vertex. It is not hard to see that $U$ will be a connected subgraph of $T$ containing $w$, that is, $U$ is a subtree containing $w$. Moreover, it is also not too hard to see that since $T$ is a tree (and hence there is a unique path between any two vertices), every vertex in $U$ except possibly for $w$ will have precisely 1 neighbor in $\langle A \backslash w\rangle$. Conversely, it is clear that if $A$ is a minimal percolating set and $S \subset A$ is a nonempty subset satisfying $U=T \backslash\langle A \backslash S\rangle$ is a subtree such that every vertex in $U$ has precisely 1 neighbor in $T \backslash U$, then $|S|=1$ (because if we add a vertex of $U$ to $A$ we will infect all of $T$ ).

We now turn to the first inequality. Let $A^{\prime} \subset T \backslash v$ be a largest minimal percolating set. Then if $A^{\prime} \cup v$ is a minimal percolating set in $T$, we are done, so suppose not. Let $X \subset A^{\prime}$ be a set of vertices such that $A^{\prime} \cup v \backslash X$ is a minimal percolating set of $A$. We see that $U=T \backslash\left\langle A^{\prime} \backslash X\right\rangle_{T}$ is a subtree of $T$ such that every vertex of $U$ except for $v$ has precisely 1 neighbor in $\backslash\left\langle A^{\prime} \backslash X\right\rangle$. Thus, $U \backslash v=\left\langle A^{\prime} \backslash X\right\rangle_{T^{\prime}}$ is a subtree of $T^{\prime}$ such that every vertex has degree 1 . Since $A^{\prime}$ is minimal, this shows $|X|=1$, which proves the first inequality.

Now let us consider the second inequality. Let $T$ be a minimal counterexample, i.e. a smallest tree such that $E(T, 2)>E(T \backslash v, 2)+1$. Let $A$ be a largest minimal percolating set of $T$. Let $w$ be the unique neighbor of $v$. If $w \in A$, then we see that $A \backslash v$ is a largest minimal percolating set of $T \backslash v$ which would prove the result, so suppose $w \notin A$. If $A \cup w \backslash v$ were minimal, we would be done, so we may suppose that $A \cup w \backslash v$ is not minimal. Let $T_{1}, \cdots, T_{k}$ be the connected components of $T \backslash\{v, w\}$, and let $A_{i}=A \cap T_{i}$. Then we see that precisely one of the $T_{k}$, say $T_{1}$, is internally spanned (there must be at least one since $A$ percolates, and there must be at most one since $A \cup w \backslash v$ is not minimal). By applying the lemma to $w \cup T_{1}$, we may find a set $A_{1}^{\prime} \subset T_{1}$ such that $w \cup A_{1}^{\prime}$ is a minimal percolating set of $w \cup T_{1}$ of size at least $\left|A_{1}\right|$. Then $w \cup A_{1}^{\prime} \cup \bigcup_{i=2}^{k} A_{i}$ is a minimal percolating set of $T \backslash v$ of size at least $|A|-1$.

Note that while the first inequality can be proved in exactly the same fashion for general $r$, the second inequality is false for general $r$. For example, consider the following tree $T$. Start with a vertex $c$, adjoin $r-1$ neighbors to it, then adjoin $r-1$ leaves to each neighbor of $c$. The vertex $c$ will be infected in any percolating set, and $c$ and the leaves will infect all of $T$, so $E(T, r)=(r-1)^{2}+1=r^{2}-2 r+2$. However, if we adjoin a single additional leaf $v$ to $c$, then we have a minimal percolating set of $T \cup v$ given by all of the leaves and all of the neighbors of $c$. Thus, $E(T \cup v, r)=r(r-1)+1=r^{2}-r+1$. 
The failure of this lemma for general $r$ prevents us from easily extending our algorithm for $E(T, 2)$ to general $r$. In fact, without this lemma (or possibly a much more lengthy analysis of various trailing subgraphs), it is difficult to imagine what a linear-time algorithm for computing $E(T, r)$ would look like.

Using Lemma 15 we can prove the correctness of our algorithm for Case C.

Lemma 16. If $\{u, v, w\} \subset T$ is a trailing $P_{2}$ with $u$ the leaf, $v$ the unique neighbor of $u$, then any set of the form $\{u, v\} \cup A^{\prime}$, with $A^{\prime}$ a largest minimal percolating set of $T \backslash\{u, v, w\}$, is a largest minimal percolating set of $T$.

Proof. It is clear that any set of the form $\{u, v\} \cup A^{\prime}$ will be a minimal percolating set of $T$, and it is also clear that all such sets will have size $E(T \backslash\{u, v, w\}, 2)+2$. Thus, it remains to show $E(T, 2) \leq E(T \backslash\{u, v, w\}, 2)+2$.

Now, it is clear that for any minimal percolating set $A$ of $T$, either $\{u, v\} \subset A$ or $\{u, w\} \subset A$. We wish to bound the size of a minimal percolating set $A$ of $T$ which contains $\{u, w\}$. If $\{u, w\} \subset A$, then $A$ consists of $u$ and a minimal percolating set of $T \backslash\{u, v\}$, so $|A| \leq 1+E(T \backslash\{u, v\}, 2) \leq 2+E(T \backslash\{u, v, w\}, 2)$ by Lemma 15. Thus, $E(T, 2)=E(T \backslash\{u, v, w\}, 2)+2$.

Finally, we prove the correctness of our algorithm for Case D.

Lemma 17. Let $T$ be a tree with a trailing straight pseudo-star with at most one leaf attached to the center vertex. Let $v$ be the center vertex, $S$ the rest of the pseudo-star, and $L \subset S$ the set of leaves. Let $T^{\prime}=T \backslash S \cup u$, where $u$ is a single leaf connected to $v$. Then given any largest minimal percolating set $A^{\prime}$ of $T^{\prime}$, the method described in Case $D$ yields a largest minimal percolating set of $T$.

Proof. The point of the proof is that, with respect to percolation, a trailing pseudo-star with at most one leaf adjacent to the center vertex "behaves exactly like" a trailing $P_{2}$. We start by describing how a trailing $P_{2}$ behaves. Given a minimal percolating set $A$ on a tree with a trailing $P_{2}$, we know that the leaf of the $P_{2}$ will be in $A$. If the $P_{2}$ is not internally spanned, then the leaf will be the only vertex of the trailing $P_{2}$ in $A$. If the $P_{2}$ is internally spanned, then one aditional vertex (namely, the other vertex of the $P_{2}$ ) will be in $A$. Moreover, if $T$ minus the $P_{2}$ is internally spanned, then the $P_{2}$ cannot be internally spanned.

Now we turn our attention to trailing pseudo-stars. First note that there will be a largest minimal percolating set of $T$ in which $v$ is not initially infected. To see this, note first that $v \cup L$ internally spans the pseudo-star, so we need only show that it is possible to choose a largest minimal percolating set $B$ of the pseudo-star with $v \notin B$. However, it is clear that we can do this (simply choose any two neighbors of $v$, including any neighbors which are leaves).

Now, since $v$ has only one neighbor outside of the pseudo-star and because the pseudostar is straight, any minimal percolating set $A$ will contain at least one neighbor of $v$ in the pseudo-star. Since $v \cup L$ infects the entire pseudo-star and because $v$ is adjacent to at most one leaf, we see that the pseudo-star will be internally spanned by $A$ if and only if 
precisely two neighbors of $v$ are in $A$. Since $v$ is adjacent to at most one leaf, this means that if $A$ is any minimal percolating set in which the pseudo-star is internally spanned and $B$ is any minimal percolating set in which the pseudo-star is not internally spanned, then $|A \cap S|$ and $|B \cap S|$ are independent of $A$ and $B$, and $|A \cap S|=|B \cap S|+1$. This is exactly the case for trailing $P_{2}$ 's, and thus, the result is proven.

This concludes the (sketch of) the proof of the correctness of the algorithm ESet.

\section{Conclusion}

Relatively little is known about extremal minimal percolating sets. We summarize here what is known and suggest several natural problems to consider. It turns out that, $m(T, r)$ is usually easier to compute than $E(T, r)$. This paper describes how to find $m$ for trees. Exact values of $m$ are known for the $n \times \cdots \times n$ grid in $d$ dimensions $[n]^{d}$ with $r=2$. In [12] Pete finds estimates for $m(G, r)$ for grids. For $E(G, r)$, we show here how to compute it for trees with $r=2$, and in [13] $E\left(Q_{n}, 2\right)$ is found exactly and shown to be $O\left(2^{n / 4}\right)$. Additionally Morris [11] showed $\frac{4 n^{2}}{33} \leq E\left([n]^{2}, 2\right) \leq \frac{n^{2}}{6}$.

This leaves many open questions related to $r$-bootstrap percolation, and the following suggestions are by no means exhaustive. We start by asking a few more questions about finite trees. For a fixed $r \geq 2$, we call a pair $(a, b)$ possible if for every $\epsilon>0$, there is a tree $T$ of size $n$ such that $\frac{m(T, r)}{n}$ is within $\epsilon$ of $a$ and $\frac{E(T, r)}{n}$ is within $\epsilon$ of $b$.

Question 18. Which pairs $(a, b)$ are possible?

For example, for $r=2$ we can use Proposition 3, Theorem 5, the fact that $m(T, 2) \leq$ $E(T, 2)$, and the fact that $E(T, 2) \leq n$ to see that any possible pair must lie in the convex hull of $(1 / 2,1 / 2),(1 / 2,3 / 4),(3 / 4,1)$ and $(1,1)$. Moreover, using Theorem 4 and the fact that $m(T, 2) \geq \ell$ we see that $E(T, 2)-\frac{m(T, 2)}{3} \leq \frac{2}{3} n$. Thus, any possible pair must lie in the convex hull of $(1 / 2,1 / 2),(1 / 2,3 / 4),(5 / 8,7 / 8)$ and $(1,1)$. On the other hand, we know that $(1 / 2,1 / 2),(1,2,3 / 4)$, and $(1,1)$ are possible using complete binary trees, the extremal example for Theorem 5 and stars, and by connecting them with short paths we know that pairs $(a, b)$ in their convex hull must also be possible. This leaves the convex hull of $(1 / 2,3 / 4),(5 / 8,7 / 8)$, and $(1,1)$ as an indeterminate region which could benefit from future study.

Another direction for future study would be to look at finer numerical invariants of trees that the number of vertices and number of vertices of degree less than $r$, and see what bounds could be proven using these invariants.

Question 19. Is there a number associated to trees (other than the number of vertices of degree less than $r$ ) which gives a stronger upper bound on $E(T, r)$ ? Does this number give us any more information about $m(T, r)$ ?

Another natural question to ask is whether or not it is possible to find an efficient algorithm to compute $E(T, r)$ for $r>2$. As we mention, this question will likely be difficult because Lemma 15 no longer holds. 
Question 20. Is there an polynomial-time algorithm for computing $E(T, r)$ for $r>2$ ? Is there an $O(n)$ algorithm?

Working in a different direction, it would also be interesting to try to apply some of the techniques of this paper to other types of graphs with relatively few edges.

Question 21. What are $m(G, r)$ and $E(G, r)$ for $G$ a unicyclic graph? Can the results for trees be extended to an even more general class of graphs?

Most of the proofs of our $m(G, r)$ algorithms carry through for general graphs. However, some of our $E(G, 2)$ procedures do not carry through, because the proof of Lemma 15 does not work for non-trees.

As Balogh, Bollobàs and Morris [5] suggest, we could also consider less sparse graphs which have more structure, such as the hypercube.

Question 22. What is $m\left(Q_{n}, 3\right)$ ? What is $E\left(Q_{n}, 3\right)$ ? Are there bounds for general $r$ ?

See the conclusion of [5] for a summary of what is known, and a conjecture for general $r$. Finally, it would be very interesting to continue the work of Morris [11] and obtain at least asymptotic results for $E(G, r)$ for grids with $r=2$ and $d \geq 2$.

Question 23. What is $E\left([n]^{d}, r\right)$ asymptotically for $r=2$ and $d \geq r$ ? Can anything be said for general $r$ ?

\section{Acknowledgements}

This research was done while I was a student at Notre Dame, as a participant in the University of Minnesota Duluth math REU, which is run by Joe Gallian. The REU was supported by the National Science Foundation and the Department of Defense (grant number DMS 0754106) and the National Security Agency (grant number H98230-06-10013). I would like to thank Joe Gallian for all of his help and encouragement. I would like to thank the program advisers Nathan Kaplan and Nathan Pflueger for all their help, especially for reading drafts of this paper. I would like to thank all of the Duluth visitors, especially Sasha Ovetsky Fradkin, Phil Matchett Wood and Ricky Liu for their help. I would also like to thank Robert Morris at Cambridge for talking to me about the problem. Finally, I would like to thank the reviewer for helping with many aspects of the paper, particularly for suggesting several of the open problems in the conclusion.

\section{References}

[1] J. Adler and U. Lev. Bootstrap percolation: visualizations and applications. Braz. J. Phys., 33:641-644, 2003.

[2] M. Aizenman and J. L. Lebowitz. Metastability effects in bootstrap percolation. J. Phys. A, 21(19):3801-3813, 1988. 
[3] J. Balogh, B. Bollobás, H. Duminil-Copin, and R. Morris. The sharp metastability threshold for $r$-neighbor bootstrap percolation. Trans. Amer. Msth. Soc., to appear.

[4] J. Balogh, B. Bollobás, and R. Morris. Bootstrap percolation in three dimensions. Ann. Probab., 37:1329-1380, 2009.

[5] J. Balogh, B. Bollobás, and R. Morris. Bootstrap percolation in high dimensions. Combin. Prob. Computing, 19:643-692, 2010.

[6] J. Balogh, Y. Peres, and G. Pete. Bootstrap percolation on infinite trees and nonamenable groups. Combin. Prob. Computing, 15:715-730, 2006.

[7] R. Cerf and E. Cirillo. Finite size scaling in three-dimensional bootstrap percolation. Ann. Probab., 27(4):1837-1850, 1999.

[8] R. Cerf and F. Manzo. The threshold regime of finite volume bootstrap percolation. Stochastic Process. Appl., 101(1):69-82, 2002.

[9] J. Chalupa, P. L. Leath, and G. R. Reich. Bootstrap percolation on a bethe lattice. J. Phys. C., 12:L31-L35, 1979.

[10] Alexander Holroyd. Sharp metastability threshold for two-dimensional bootstrap percolation. Probab. Theory Related Fields, 125(2):195-224, 2003.

[11] Robert Morris. Minimal percolating sets in bootstrap percolation. Electron. J. Combin., 16(1):\#R2, 20pp, 2009.

[12] Gabor Pete. Disease processes and bootstrap percolation. Thesis for diploma at the Bolyai Institute, József Attila University, Szeged, 1997.

[13] Eric Riedl. Largest minimal percolating sets in hypercubes under 2-bootstrap percolation. Electron. J. Combin., 17(1):\#R80, 13pp, 2010. 